\title{
Can mercury stable isotopes aid monitoring and effectiveness evaluation of the Minamata Convention on Mercury?
}

\author{
SAE YUN KWON
}

Pohang University of Science and Technology

Presenting Author: saeyunk@postech.ac.kr

While multiple measurement and modeling techniques are being considered for a global monitoring and effectiveness evaluation of the Minamata Convention on Mercury (MC), the potential utility of mercury isotopes remains a question. Since the early 2000 s, mercury isotope ratios have been measured in natural environmental samples to distinguish between the relative influence of various anthropogenic and natural mercury sources and to understand complex processes occurring in ecosystems. Assessing whether anthropogenic mercury sources governed under the $\mathrm{MC}$ is decreasing in the environment is the primary objective of the global monitoring and effectiveness evaluation. In this regard, mercury isotopes can complement existing measurement and modeling techniques by providing source- and process-based information used to interpret temporal trends in environmental mercury levels. In an earlier work, we compiled a dataset from the literature to understand patterns of mercury isotope ratios in various environmental samples (samples from the atmosphere, terrestrial and aquatic environments) and to identify sources and processes driving these patterns in the environment. We illustrate that atmospheric samples including total gaseous mercury (TGM) and precipitation are particularly useful for identifying the degree of anthropogenic mercury source influence and for assessing spatially variable processes leading to mercury transport and deposition to land surfaces. While the tissues of biota (fish, mammals) are being considered as a proxy for the effectiveness evaluation, the presence of internal mercury isotope fractionation and exposure to multiple dietary mercury sources make it difficult to establish a largescale isotopic pattern. Through both experimental and field studies, we illustrate how mercury isotope ratios in biota can be used to trace anthropogenic mercury sources at mercury hotspots and generate ecosystem and human health-relevant information. 